\title{
The influence of restricted orientation rearing on map structure in primary visual cortex
}

\author{
Clare E. Giacomantonio ${ }^{\text {a }}$, Michael R. Ibbotson ${ }^{\text {b }}$, Geoffrey J. Goodhill ${ }^{\text {a,c,* }}$ \\ a Queensland Brain Institute, The University of Queensland, St Lucia, QLD 4072, Australia \\ ${ }^{\mathrm{b}}$ Visual Sciences Group and Australian Research Council Centre of Excellence in Vision Science, Australian National University, Canberra, ACT 2601, Australia \\ c School of Mathematics and Physics, The University of Queensland, St Lucia, QLD 4072, Australia
}

\section{A R T I C L E I N F O}

\section{Article history:}

Received 20 April 2009

Revised 11 December 2009

Accepted 15 December 2009

Available online 24 December 2009

\section{Keywords:}

Orientation map

Ocular dominance

Visual deprivation

Visual plasticity

Computational model

\begin{abstract}
A B S T R A C T
Visual experience is critical to the development of the structure of the primary visual cortex and, in turn, normal functional vision. The primary visual cortex contains maps of multiple features of the visual input, and these maps are characterised by specific types of geometric relationships. Manipulations of the visual environment during development in animals such as ferrets, cats and monkeys provide an opportunity to probe the rules governing map formation via their effect on these relationships. Here we use a computational model of map formation based on dimension-reduction principles to predict the effect on map relationships of presenting only a single orientation to one eye and the orthogonal orientation to the other eye. Since orientation preference and ocular dominance are now tightly coupled one might expect orientation and ocular dominance contours to lose their normally orthogonal relationship and instead run parallel to each other. However, surprisingly, the model predicts that orthogonal intersection can sometimes be preserved in this case. The model also predicts that orientation pinwheels can migrate from the centre to the borders of ocular dominance columns, and that the wavelengths of the ocular dominance and orientation maps can become coupled. These predictions provide a way to further test the adequacy of dimension reduction principles for explaining map structure under perturbed as well as normal rearing conditions, and thus allow us to deepen our understanding of the effect of the visual environment on visual cortical development.
\end{abstract}

(c) 2009 Elsevier Inc. All rights reserved.

\section{Introduction}

Visual experience is crucial to the development of the primary visual cortex (V1), and abnormal development can profoundly affect functional vision. For example, amblyopia is a common condition in humans that is characterised by a loss of visual acuity in one eye that is not caused by physical defects in the eye or any disease. The visual defects of human amblyopes have been reproduced in animal models through various manipulations of visual input such as monocular deprivation (Mitchell and Sengpiel, 2009). Electrophysiological and imaging studies of animals have also revealed the structural changes resulting from monocular deprivation that cause loss of acuity. More generally, manipulations of the visual environment of animals during their development have been used extensively to probe how environment and experience influence the structure and function of the visual system.

Neurons in V1 respond preferentially to stimuli with particular combinations of features. Amongst other features, neurons tend to prefer stimuli of a particular orientation (orientation preference, OP)

\footnotetext{
* Corresponding author. Queensland Brain Institute and School of Mathematics and Physics, The University of Queensland, St Lucia, QLD, 4072, Australia. Fax: +61 733466301. E-mail address: g.goodhill@uq.edu.au (G.J. Goodhill).
}

presented in one eye (ocular dominance, OD). In many mammals including cats (Crair et al., 1997a; Müller et al., 2000), monkeys (Blasdel, 1992a,b; Bartfeld and Grinvald, 1992; Obermayer and Blasdel, 1993) and ferrets (Müller et al., 2000), feature preferences co-exist in such a way that each combination of features is represented in the cortex (good coverage), while adjacent neurons across the surface of the cortex have similar response properties (good continuity) (Swindale, 1991). This results in feature maps, complex but stereotypical spatial patterns of response properties. Moving perpendicular to the cortical surface, cells tend to form columns with similar response properties (Hubel and Wiesel, 1977), and it is therefore often a good approximation to treat feature maps as two-dimensional.

These feature maps often have characteristic geometric relationships to each other. For instance, OP contours tend to intersect OD column borders perpendicularly in normal animals (Bartfeld and Grinvald, 1992; Blasdel, 1992b; Obermayer and Blasdel, 1993; Hübener et al., 1997; Kim et al., 1999; Müller et al., 2000). In addition, orientation pinwheels tend to be located at the centre of OD columns (Bartfeld and Grinvald, 1992; Obermayer and Blasdel, 1993; Hübener et al., 1997). However, manipulating the statistics of visual inputs in different rearing regimes challenges map formation and the relationships between maps. Such experiments probe the degree to which the 
structure of visual maps is intrinsic or influenced by sensory experience. While there is evidence that some aspects of map structure have an inherited component (Kaschube et al., 2002, 2003), other work has also demonstrated that visual inputs affect the structure of the relevant cortical map. For example, single orientation rearing causes the OP columns of the over-represented orientation to expand (Sengpiel et al., 1999; Tanaka et al., 2006).

"Dimension reduction" models have been successful at predicting and reproducing the structure of feature maps in V1 and the relationships between maps (Durbin and Mitchison, 1990; Goodhill and Willshaw, 1990; Obermayer et al., 1992; Erwin et al., 1995; Swindale, 1996; Goodhill et al., 1997; Swindale and Bauer, 1998; Goodhill and Cimponeriu, 2000; Carreira-Perpiñán et al., 2005; Yu et al., 2005; Farley et al., 2007). These algorithms map a highdimensional feature space based on visual inputs onto a twodimensional surface representing V1 under the competing constraints of good continuity and coverage. The models also reproduce the consequences of several experimental manipulations, including monocular deprivation and single orientation rearing (Goodhill and Willshaw, 1994; Carreira-Perpiñán et al., 2005).

An experimental manipulation that has not yet been explored by modelling is when individuals experience only horizontal lines in one eye and only vertical lines in the other eye. Several older experimental studies examined the effect on cat V1 of this rearing regime, but only with single-unit recordings (Hirsch and Spinelli, 1970, 1971; Stryker et al., 1978). These recordings revealed that neurons that responded to one eye were selective for vertical orientation while neurons that responded to the other eye were selective for horizontal orientations. However, whether the OP map altered to match the OD map or vice versa was not determined. Investigations of the consequences of this rearing paradigm using more modern optical imaging techniques are so far preliminary, and have only been presented in abstract form (Tani and Tanaka, 2008). In particular, the geometric relationships between the OD and OP maps arising from this rearing regime have not been fully explored, nor have the effects of the strength and duration of the orientation-restricted rearing on the resulting maps been investigated.

Here, we use an established model to explore this rearing regime and make novel predictions about joint OP and OD map development. Our results not only agree with the data from single-electrode recordings, but also extend previous work by visualising in full the effect on cortical maps as well as analysing the geometric relationships between maps. We predict that map development under restricted orientation rearing in the model proceeds in two stages. In the first stage, OP columns for the over-represented orientations form and precipitate the formation of OD columns perfectly aligned with the OP columns. In the second stage, other OP columns form at the OD column boundaries. This change in the OP map causes a concomitant rearrangement of the OD map and a change in the geometric relationships between the two maps. These results present a further opportunity to test the predictions of models based on trading off coverage and continuity constraints for explaining activitydependent visual map development.

\section{Methods}

\section{The elastic net algorithm}

Many different computational models for visual map development have been proposed (Erwin et al., 1995; Swindale, 1996, 2003; Goodhill, 2007; Simpson et al., 2009). In most of these, including the elastic net used here, a set of visual inputs is mapped onto a twodimensional array of cells representing V1. Many of these models have been shown to produce maps which closely match much of the experimental data regarding the structure of cortical maps and the relationships between maps. The elastic net in particular has reproduced the experimentally observed effects on map structure of single orientation rearing (Carreira-Perpiñán et al., 2005), monocular deprivation (Goodhill and Willshaw, 1994; Carreira-Perpiñán et al., 2005) and angioscotoma representation (Giacomantonio and Goodhill, 2007).

Here we use the low-dimensional version of the elastic net model which treats each combination of input features, such as an oriented edge at a particular position in space, as a point in a 'feature' space. A net of points representing cortical cells occupies this feature space and the position of each cortical cell in this high-dimensional space $\mathbf{w}_{j}$ represents its full receptive field, e.g. ( $x$-position, $y$-position, ocularity, orientation preference, orientation selectivity). This abstraction speeds up computation while still producing results in good agreement with the biological data (Erwin et al., 1995; Swindale, 1996).

At each iteration, feature points which uniformly sample the feature space are presented to the elastic net. The same set of feature points are presented simultaneously at each iteration (batch mode). This method speeds up the computation and generates smooth maps from only a relatively small number of feature points. It has previously been shown to generate maps which closely resemble those seen biologically (Goodhill and Willshaw, 1990, 1994; Goodhill and Cimponeriu, 2000; Carreira-Perpiñán et al., 2005; Giacomantonio and Goodhill, 2007). The 'activity', $\Phi$, of each cortical cell $\mathbf{w}_{j}$ in response to the input feature $\mathbf{v}_{i}$ is related to the distance in feature space between the cortical point and the feature point, scaled by the parameter $K$ :

$\Phi\left(\left\|\mathbf{v}_{i}-\mathbf{w}_{j}\right\|, K\right)=\exp \left(-\frac{\left\|\mathbf{v}_{i}-\mathbf{w}_{j}\right\|^{2}}{2 K^{2}}\right)$.

These activities are normalised so that each input feature elicits the same amount of activity in the cortex:

$n_{i j}=\frac{\Phi\left(\left\|\mathbf{v}_{i}-\mathbf{w}_{j}\right\|, K\right)}{\sum_{p} \Phi\left(\left\|\mathbf{v}_{i}-\mathbf{w}_{p}\right\|, K\right)}$.

The receptive field of each cortical cell $\mathbf{w}_{j}$ is then adapted towards each input feature by an amount proportional to the degree $n_{i j}$ to which it responded to that feature:

$\Delta \mathbf{w}_{j}=\alpha \sum_{i} n_{i j}\left(\mathbf{v}_{i}-\mathbf{w}_{j}\right)+\beta K \sum_{j^{\prime} \in N(j)}\left(\mathbf{w}_{j^{\prime}}-\mathbf{w}_{j}\right)$.

Here, $N(j)$ refers to the set of points in the cortical sheet that are neighbouring cortical point $j$. The first term is a summation of the influence of all input features on cortical cell $j$ so that cell $j$ adapts a little to each input feature simultaneously. The second term in Eq. (3) models the effect of lateral interactions which move neighbouring cortical points towards each other in feature space to keep the receptive fields of neighbouring cortical cells similar to each other (Dayan, 1993; Yuille et al., 1996; Carreira-Perpiñán and Goodhill, 2004).

The mapping procedure attempts to improve a trade-off between good coverage and good continuity, two properties of real cortical maps (Swindale, 1996). The cortical points must adequately sample the feature space in order to avoid perceptual blind spots (good coverage) and neighbouring cortical points must be close together in feature space to produce the smooth maps observed in V1 (good continuity). The relative strengths of the coverage and continuity terms are controlled by the ratio $\alpha / \beta$.

$K$ is reduced over the course of a simulation. As a result, the effect of each feature point on cortical points is increasingly local (Eq. (3), first term). At each iteration, every feature point pulls on every cortical point, contributing a component to the net displacement 
of each cortical point $\Delta \mathbf{w}_{j}$, but the size of this component changes with the value of $K$. Initially, when $K$ is large, there is only a small decrease in the pulling force of each feature point with distance (Eq. (1)) so that each feature point shares its influence among many cortical points. As $K$ decreases, the decrease with distance becomes much sharper so that each feature point influences an increasingly local subset of cortical points. The normalisation in Eq. (2) ensures that the net movement caused by each feature point on the set of all cortical points remains the same at each value of $K$. In this way, as $K$ is reduced, each feature point pulls more strongly on closer cortical points at the expense of pull on more distant cortical points. The overall effect of reducing $K$ on the first term of Eq. (3) is that cortical points become more selective for particular combinations of input features. Reducing $K$ also affects the second term of Eq. (3) in that the attraction between neighbouring cortical points decreases.

The overall effect is that the cortical points tend to move closer to the feature points to which they are already closest, while still remaining close in feature space to their cortical neighbours. In terms of cortical mapping, this translates into cortical points becoming more selective for a particular combination of input features, while keeping the receptive fields of neighbouring cortical points similar so that maps are locally smooth.

\section{The feature space and training regime}

The simulations in this paper mapped a grid of cortical points to a feature or stimulus space of five orthogonal dimensions: two dimensions of visual field, one dimension of OD and one each of OP and orientation selectivity. This gave us co-existing cortical maps for retinotopy, OD, and $\mathrm{OP}$ weighted by orientation selectivity.

The feature space was populated by a regular array of feature points representing different stimuli. The visual field projection of the feature points was a $N_{x} \times N_{y}$ grid in the square $[0,1] \times[0,1]$. In the OD dimension there were points at $-l$ and $l$. Orientation preference and selectivity of each point were in polar coordinates. $N_{O P}$ values of orientation were arranged uniformly around a ring of radius $r$, from $[-\pi / 2, \pi / 2]$ as orientation is $\pi$-periodic. The relative value of $r$ and $l$ dictates the relative timing of development of the OP and OD maps (Hoffsümmer et al., 1995, 1996; Goodhill and Cimponeriu, 2000). We chose values such that the OP map formed before the OD map, as current experimental evidence indicates occurs in cats (Hubel and Wiesel, 1963; Blakemore and Van Sluyters, 1975; Crair et al., 1998, 2001). (However, for an alternative point of view see Crowley and Katz (2000) and Katz and Crowley (2002).

In the simulations presented here, the grid of cortical points was $128 \times 128$ in size. It started from a random initial configuration in the orientation and ocular dominance dimensions. The initial positions of cortical points in the visual field dimensions had a global topographic bias. Points were spaced uniformly $1 /(128-1)=0.0079$ units apart along the $x$ and $y$ dimensions and then shifted random distances drawn from a uniform random distribution in the interval $(-0.025$, 0.025). Other parameters took the following values: $N_{x}=N_{y}=20$, $l=0.05, N_{O P}=6$ and $r=0.08$.

For all simulations, the annealing parameter $K$ was reduced (annealed) exponentially from 0.2 to 0.01 at a rate of 0.9925 per iteration relative to the previous iteration over the course of the 400 iterations. All feature points were presented simultaneously at every iteration (batch mode) so that the sum in the first term of Eq. (3) is over all 4800 feature points at each iteration. The continuity constant, $\beta$, was 10 . The coverage constant, $\alpha$, varied between orientations to simulate the over-representation of selected orientations (for further explanation see the next section, Restricted orientation rearing). For each set of $\alpha$-values, 10 maps were simulated starting from a different random initial configuration of the cortical net.

\section{Restricted orientation rearing}

Different single orientations in each eye were simulated in a similar manner to the monocular deprivation and single orientation rearing simulations in Carreira-Perpiñán et al. (2005). In the normal case, the coverage constant $\alpha=\alpha_{\mathrm{N}}=1$ for all feature points so that each combination of features has an equal effect on the cortical net. To enhance particular orientations in each eye, we redefined the coverage constant as a vector $\alpha$ so that each feature point $\mathbf{v}_{i}$ had an individual element $\alpha_{i}$ associated with it. We increased the effect of a subset of feature points on the cortical points by setting $\alpha_{i}>1$ for each $\mathbf{v}_{i}$ corresponding to one orientation in one eye and each $\mathbf{v}_{i}$ corresponding to the orthogonal orientation in the other eye.

In order to maintain the same overall trade-off between coverage and continuity for all simulations, we compensated for raising $\alpha_{i}$ for the over-represented orientations by decreasing $\alpha_{i}$ for the non-overrepresented orientations. The sum of $\alpha_{i}$ over all orientations in each eye remains constant. For each set of simulations we chose the ratio, $R_{\mathrm{b}}$, of $\alpha_{i}$ for the over-represented orientations to $\alpha_{i}$ for the non-overrepresented orientations and calculated the values of $\alpha_{i}$ as follows:

$$
\begin{gathered}
\alpha_{i}^{\text {other orientations }}=\frac{N_{O P} \times \alpha_{N}}{R_{b}+N_{O P}-1} \\
\alpha_{i}^{\text {boosted orientations }}=R_{b} \times \alpha_{i}^{\text {other orientations }} .
\end{gathered}
$$

Simulation results are presented for $R_{\mathrm{b}}=1$ (normal case, $\alpha=1$ for all orientations) and $\left\{R_{\mathrm{b}} \in \mathbb{Z} \mid 2 \leq R_{\mathrm{b}} \leq 10\right\}$. The larger the ratio $R_{\mathrm{b}}$, the greater the strength of over-representation. This could simulate an increasing strength of the goggle filters or an increasing number of hours per day that an individual animal wears goggles that alter the visual environment.

Orientations in the model are represented abstractly as the position in the OP dimension of a high-dimensional feature space. Consequently, the allocation of specific angles to the points in the OP dimension is arbitrary. Throughout the rest of the paper, we will refer to an over-representation of horizontal contours in the right eye and vertical contours in the left eye; however, the results apply equally to any two orthogonal angles presented in either eye.

\section{Analysis of maps}

The results of the elastic net simulations are a set of retinotopic, OD and OP maps where at each pixel the full receptive field ( $x$ - and $y$ position, ocularity, OP and orientation selectivity) is defined.

We focused on examining the global structure of maps. The OD/OP intersection angle (Fig. 2), number of OP pinwheels and distance from pinwheels to closest OD border (Fig. 4) were calculated using the procedures described by Carreira-Perpiñán et al. (2005). Intersection angles between OD and OP contours in each map were calculated by averaging over all pixels, but weighting the intersection angle at each pixel by the product of OD and OP gradient steepness, $\|\nabla O P\| \times\|\nabla O D\|$. This is consistent with previous map analyses (Bartfeld and Grinvald, 1992; Obermayer and Blasdel, 1993; Hübener et al., 1997; Löwel et al., 1998; Yu et al., 2005) and effectively removed areas of constant OP or constant OD or both, since gradient direction is not very well defined in these areas. The resulting distributions of weighted intersection angles in Fig. 2 are referred to as histograms.

The mean wavelength of each map (Fig. 3) was calculated from the two-dimensional discrete Fourier transform of the map

$\lambda=\sum_{n=1}^{128 \times 128} P_{n} / k_{n}$

where $P_{n}$ is the power of the $n$-th pixel in the two-dimensional Fourier transform and $k_{n}$ is the wave-number corresponding to that 
pixel. We calculated the OP wavelength from the angle maps (where pixel colour represents OP), not the polar maps (where, in addition to $\mathrm{OP}$, pixel intensity is used to represent orientation selectivity). In most cases, the power spectrum was approximately isotropic and annular for both the OD and OP maps, similar to the example seen in Fig. 3F. However, in stage two for $R_{\mathrm{b}}>1$, the power spectra of the OP maps became more diffuse, similar to the example in Fig. 3G and so the maps are less well-defined by a mean wavelength.

Each statistic is only included in Figs. 2-4 after the relevant map had formed. We defined that a map had formed along the OD or OP dimension when a least one point of the elastic net was over $20 \%$ of the way to the maximum position along that dimension. That is, the OD map had formed when at least one point on the elastic net was at an absolute distance of $0.2 l$ along the OD dimension, and the OP map had formed when a least one point on the elastic net was at a distance of $0.2 r$ along the orientation selectivity dimension.

\section{Results}

Experimentally, single-electrode recordings in cats have found that presenting horizontal lines in the right eye, and vertical lines in the left eye, produces V1 neurons that are responsive to right eye input and horizontal orientation or left eye input and vertical orientation (Hirsch and Spinelli, 1970, 1971; Stryker et al., 1978). There are a number of possible ways in which maps could change to produce these results. The OP map could rearrange to align with the OD map, or vice versa. In the extreme case that all left-eye V1 neurons only respond to vertical lines and right-eye neurons to horizontal lines, it follows that the OP contours will coincide with OD column borders and run parallel, and the wavelengths of the two maps will match. As OP contours normally tend to intersect OD borders perpendicularly, one could hypothesise that if OP columns for the non-over-represented orientations are present, OP contours would tend to intersect at progressively smaller angles for stronger over-representations. If other OP columns are present, overrepresented OP columns could expand at the expense of those OP columns, squeezing them together. In this case, the OP wavelength would be similar to the normal case, but pinwheels would be closer to OD borders. Alternatively, if the width of the non-overrepresented OP columns remained constant, then OP wavelength would increase with over-representation and the absolute pixel distance from pinwheels to the closest OD column border would remain constant. We simulated the restricted orientation rearing regime using the elastic net model of feature map development (Durbin and Willshaw, 1987; Durbin and Mitchison, 1990; Goodhill and Willshaw, 1990). This allowed us to distinguish which possibilities result from development under the joint constraints of coverage and continuity.

\section{The appearance of maps and their developmental time-line}

We analysed $n=10$ maps as they evolved in time for each of 10 different strengths of over-representation of horizontal lines in one eye and vertical lines in the other eye. In the normal case in the elastic net, all OP columns form at the same time and the timing of OD and OP map development are controlled independently by different parameters (Fig. $1, R_{\mathrm{b}}=1$, where $R_{\mathrm{b}}$ is the strength of the over-represented orientations relative to the others). We found that the restricted orientation rearing regime causes the development of the OD and OP maps to become coupled and to occur in two distinct stages, with a brief transition between stages. The stages are defined by visual inspection of the maps and the relationships between maps.

Stage one is characterised by the presence of only vertical and horizontal OP columns that form earlier than in the normal case (Fig. 1, stage 1). The early formation of OP columns causes OD columns to segregate earlier than usual, at the same time as the OP

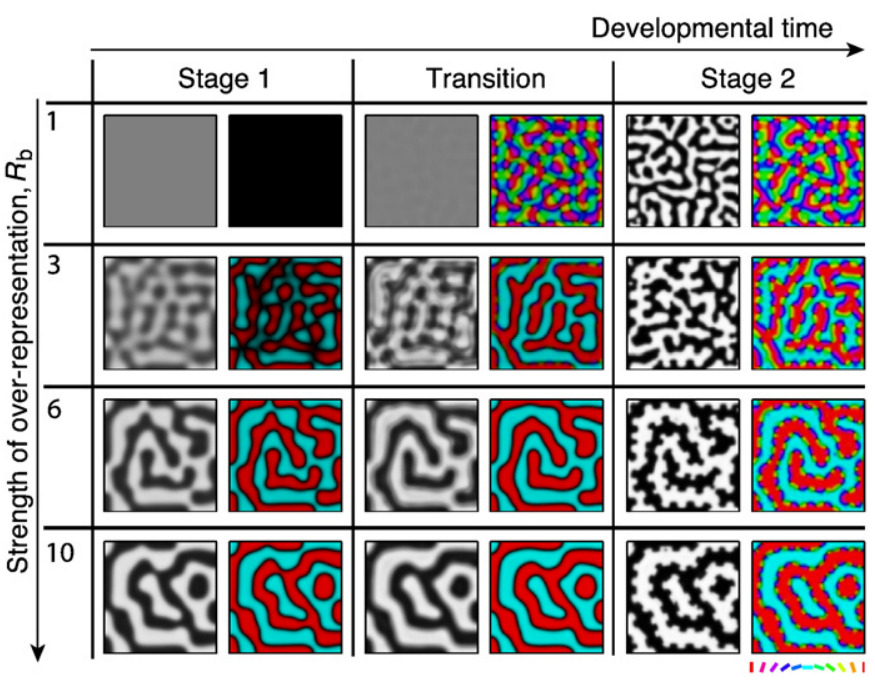

Fig. 1. Example OD and OP maps from different stages of development. The left map of each column shows an OD map where greyscale indicates the proportion of input each cell receives from the left and right eyes. Black indicates that all connections come from the left eye and white from the right eye. OP maps on the right of each column are polar; the colour of each pixel indicates the OP of the cell according to the key in the bottom right, the brightness indicates the orientation selectivity strength. Stage 1: During this stage, OP maps contain only the over-represented orientations and the structure of OD maps matches closely. OP columns for the over-represented orientations coincide with the OD column for the eye in which each orientation was presented. Transition: In the transition, OP columns for other orientations begin to form and binocular zones appear at the edges of the OD columns. Stage 2: In stage two, narrow columns for the non-overrepresented orientations appear at the OP column borders. Stronger orientation overrepresentation results in less cortical area devoted to processing other orientations and the maintenance of stronger coupling between the OD and OP maps. These example maps are taken at $K=0.048$ for stage one, $K=0.039$ for the transition and $K=0.031$ for stage two.

columns. OD maps form in such a way that OP columns for the overrepresented orientations are aligned precisely with the correct eye's OD columns. That is, the left eye OD columns (black) align with the vertical OP columns (red), while the right eye OD columns (white) align with the horizontal OP columns (cyan). As the strength of over-representation increases, stage one lengthens in duration by beginning earlier and ending slightly later.

By stage two, OP columns for oblique orientations form at the common borders of the horizontal and vertical OP columns and the OD columns (Fig. 1, stage 2). In this stage, each eye's OD columns contain a complete set of orientation columns, although each OP column does not occupy an equal cortical area. The proportion of cortical area occupied by the over-represented OP columns increases with stronger over-representation at the expense of the non-overrepresented OP columns. In the left eye OD columns, the number of vertical-preferring cells increases while in the right eye OD columns, the number of horizontal-preferring cells increases. Within each eye's OD columns, there are equal areas of cortex responsive to the nonover-represented orientations, indicating that the non-over-represented orientations are penalised equally. Note that in the normal case $\left(R_{\mathrm{b}}=1\right)$, the maps do not pass through stage one, but proceed directly to an arrangement most similar to stage two, with all OP columns present.

Between the two stages, there is a brief transition where the OP columns for the non-over-represented orientations are just forming. During this time, the OD map changes concomitantly via binocular regions forming at the OD column borders (Fig. 1, transition). For low over-representation strength, the OD map morphs significantly during this time (compare Fig. $1, R_{\mathrm{b}}=3$, stage 1 to stage 2 ). For stronger over-representation, the overall layout of the OD map does not change. During the transition, the geometric relationships are intermediate between those of the two stages. 
$O D$ and $O P$ column intersection angle

In stage one, because only OP columns for the over-represented orientations are present, OP contours tend to be parallel to OD column borders (Fig. 2A). Once the additional OP columns have formed in stage two, the tendency for the OP contours and OD borders to intersect perpendicularly is surprisingly robust (Fig. 2C). This tendency is stronger for less severe over-representations, but becomes pronounced even for strong over-representations at late stages of development. This major change in the relationship between the two maps occurs quickly during the transition (Figs. 2B, D).

The change in intersection angle occurs because of how the OD map morphs during the transition stage while the additional OP columns are forming. The contours can intersect perpendicularly even for strong over-representations because, by stage two, the OD columns "zig-zag" across the newly formed OP columns, around the newly created pinwheels (Fig. 2F).

\section{$O D$ and $O P$ map wavelength}

The wavelengths of the OD and OP maps were calculated using discrete Fourier transforms (see Methods). The wavelength of an OP map calculated using this method is a set of OP columns representing one cycle of orientations.

Figs. 3A-C shows how the OD and OP map wavelengths change as the strength of over-representation increases, at different points in development. Figs. 3D and E show the same data plotted against development time. In stage one, both OD and OP map wavelengths remain relatively constant (Fig. 3A). During this time, because OD column formation is precipitated by OP column formation and the two maps are tightly linked in structure, the wavelengths of the two maps are approximately equal.

In the transition stage, the relationship between the wavelengths of the two maps changes depending on the strength of overrepresentation (Figs. 3B, D, E). For strong over-representations, the OD map wavelength remains approximately constant (Fig. 3D, red line) while the OP map wavelength decreases (Fig. 3E, red line). For weaker over-representation, both the OD and OP map wavelengths decrease (Fig. 3D, E, yellow and green lines).

The changes in the map wavelengths during the transition mean that, by stage two, for strong over-representations, the wavelength of the OP map is roughly half its value in stage one (Fig. 3E, red line), and therefore half the OD map wavelength (Fig. 3C, $R_{\mathrm{b}}=10$ ). Even though the patterns of columns in the OD and OP maps still look very similar to each other in stage two (see Fig. $1, R_{\mathrm{b}}=6,10$, stage 2), the OP map wavelength is half the OD map wavelength because of the extra OP columns that form at the OD column borders during the transition. Due to these emerging OP columns, by stage two there is a complete set of OP columns within each left eye OD column, and another within each right eye OD column (although the width of the OP columns is not equal). Hence, according to the definition of one wavelength as a complete cycle of columns, the wavelength of the OP map is half that of the OD map. For weak over-representations, the wavelengths of the two maps are not as tightly coupled by stage two because the OD map undergoes a more significant rearrangement during the transition (Figs. 3D, E, yellow and green lines).

An alternative definition of wavelength for the OP maps with an over-representation in stage two (Fig. $1, R_{\mathrm{b}}=3,6,10$ ) is that one
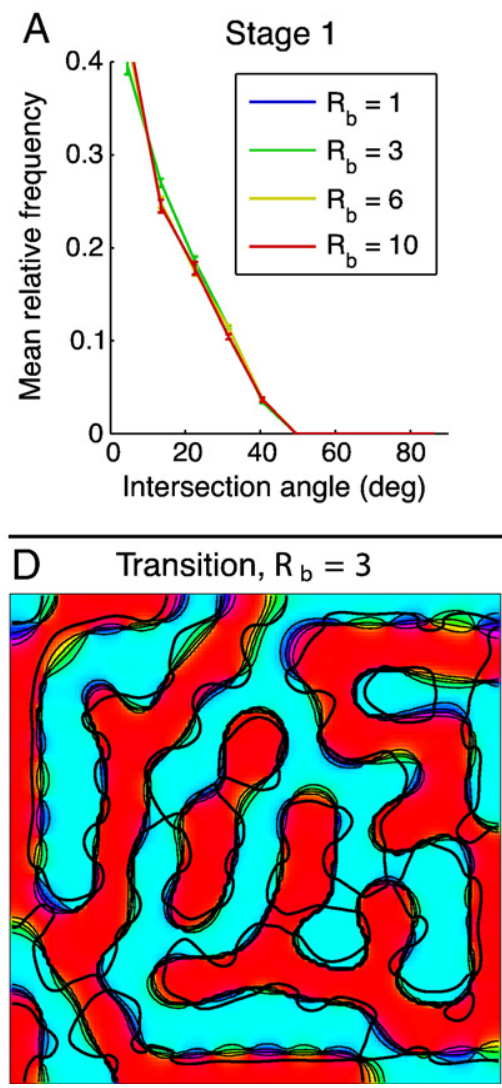
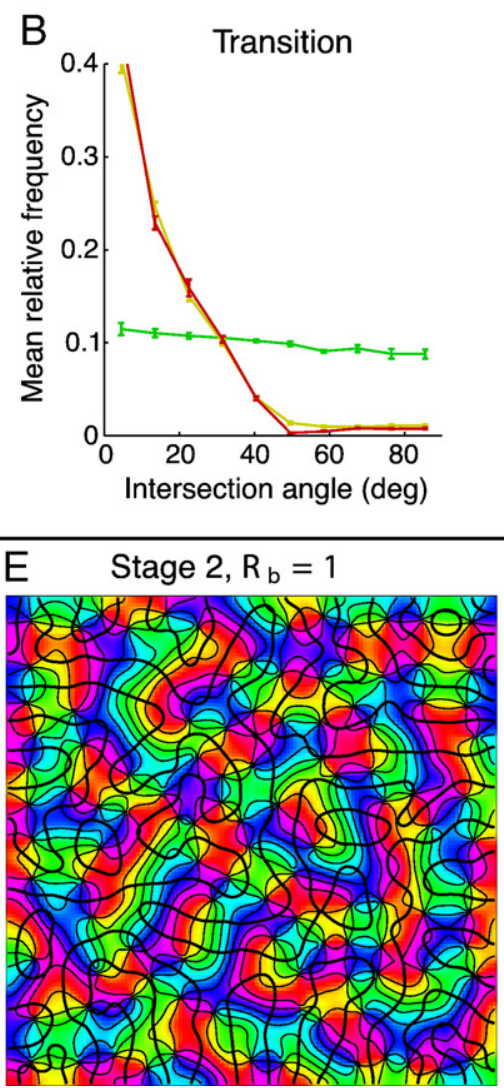
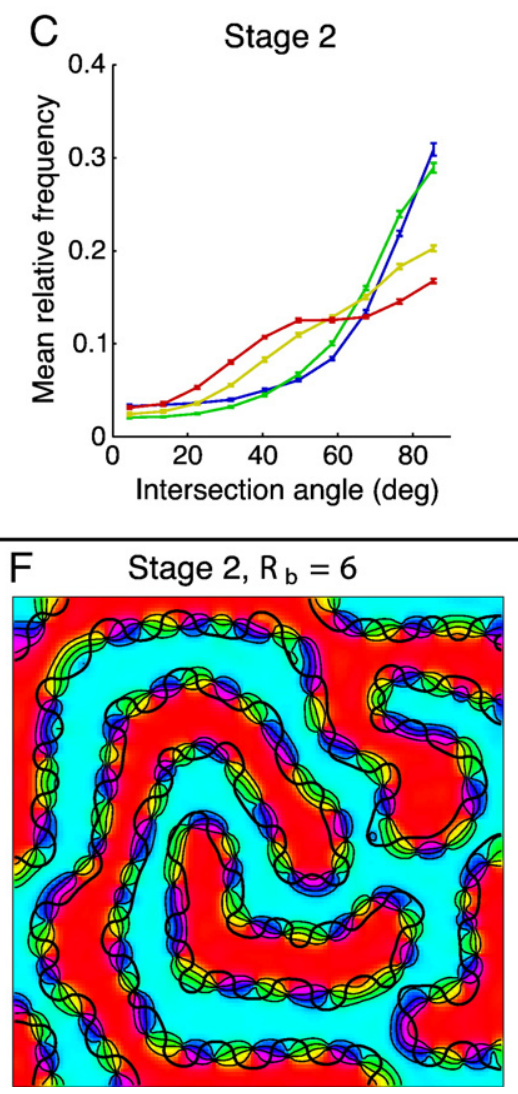

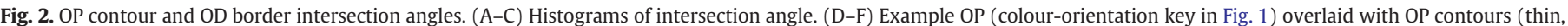

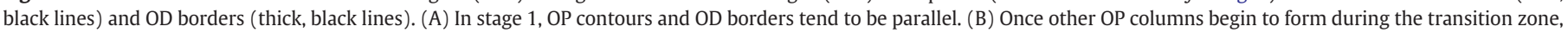

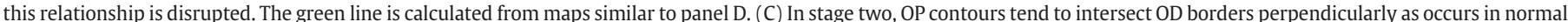

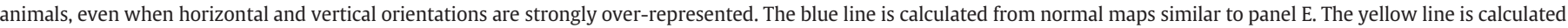

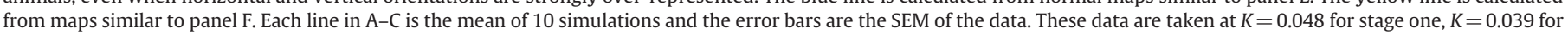
the transition and $K=0.031$ for stage two. 
A

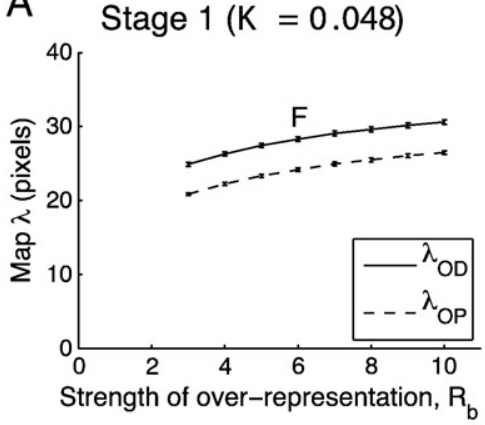

B Transition ( $\mathrm{K}=0.039)$

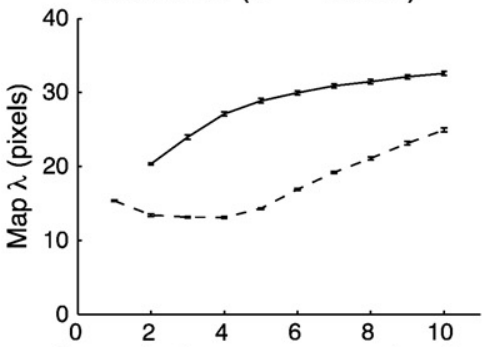

Strength of over-representation, $\mathrm{R}_{\mathrm{b}}$
C Stage $2(K=0.031)$

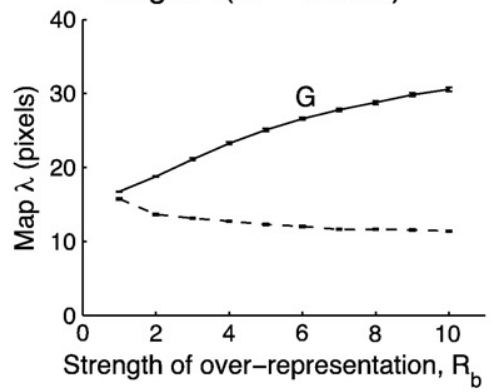

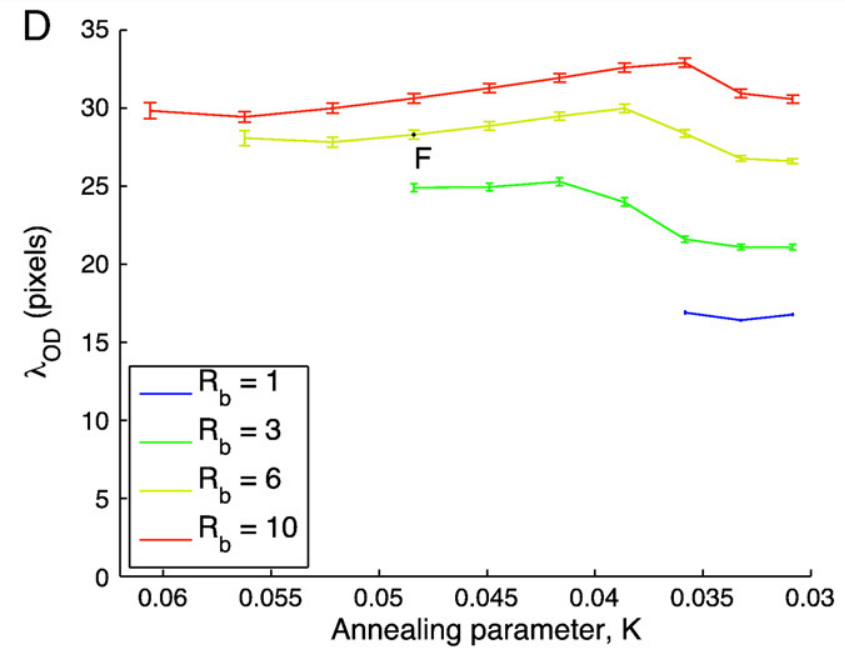
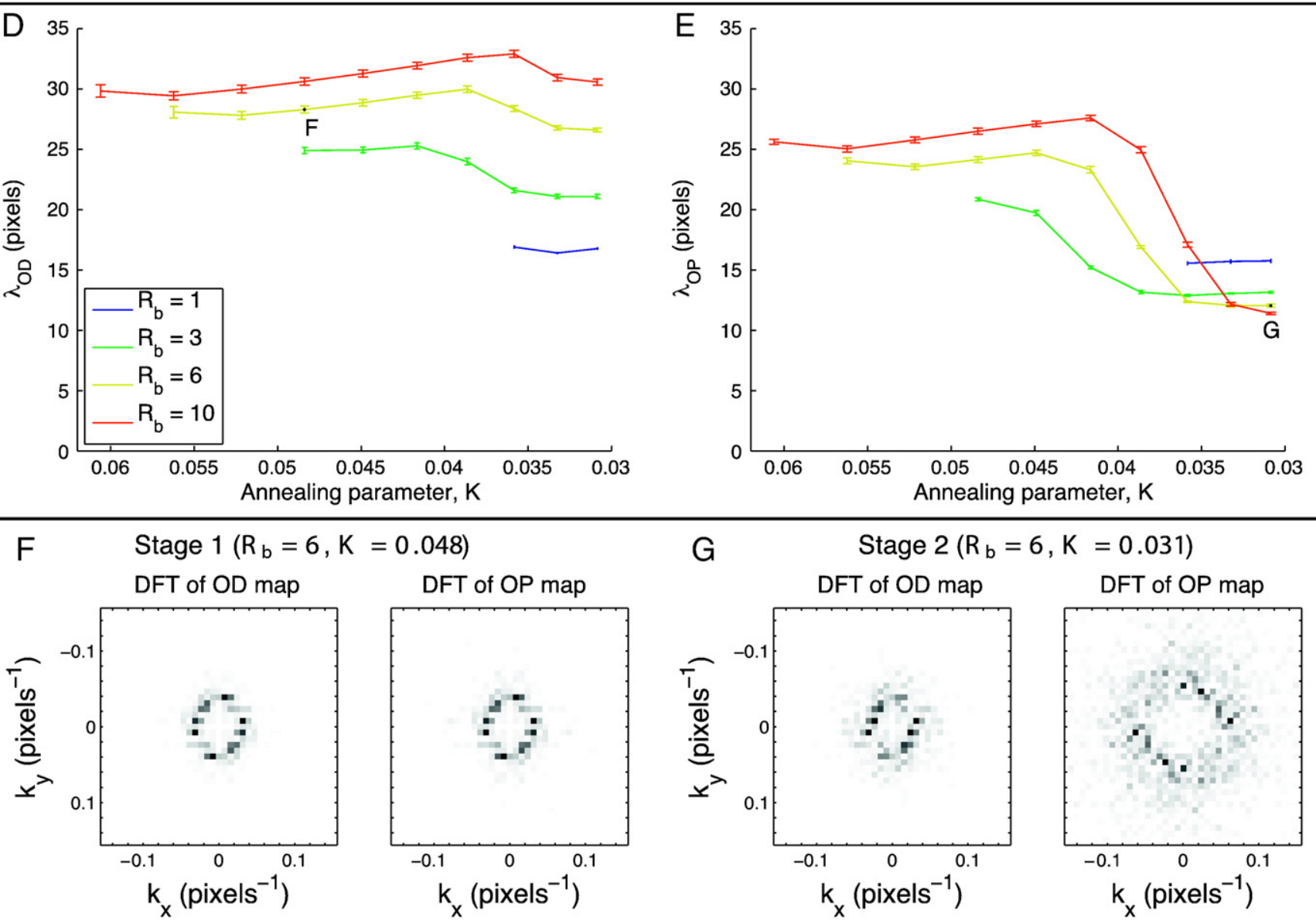

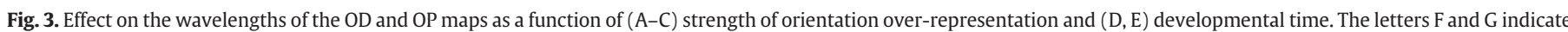

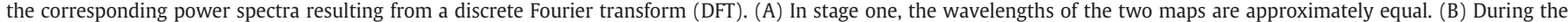

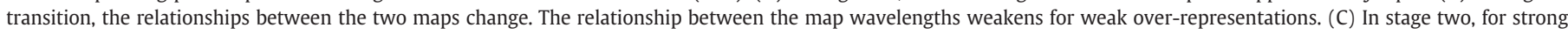

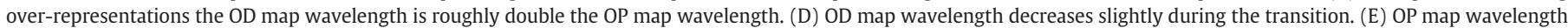

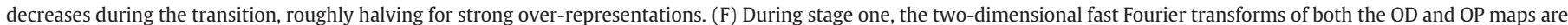

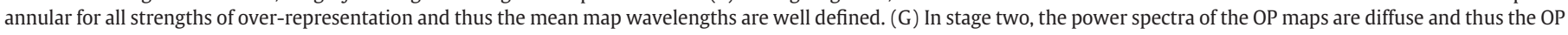

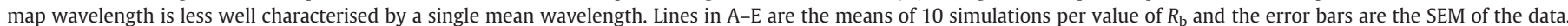

wavelength comprises one wide horizontal (cyan) column, a set of narrow columns, one wide vertical (red) column and another set of narrow columns. Under this definition, the OP map wavelength would be twice the value calculated from the Fourier transform and OP and OD maps would have the same wavelength in stage two. This alternative definition, while valid for the stage two maps, cannot be applied uniformly to all OP maps in our set. In particular, a different definition of wavelength and method of calculation would have to be used for the normal compared to the restricted orientation rearing OP maps, and for OP maps in stage one compared to stage two.

It should be noted though that the decrease in OP map wavelength during the transition does not require rearrangement of the entire $\mathrm{OP}$ or OD map between stages one and two. The wide OP columns for the over-represented orientations are largely unchanged and still largely coincide with the relevant eye's OD columns.

Another point to note is that the wavelength of the OP map is much less well-defined in stage two for maps with an overrepresentation of cardinal OPs as power in the two-dimensional discrete Fourier transform is diffuse (Fig. 3G). For all OD maps and for stage one OP maps, the power spectrum is annular and thus the maps are well characterised by the mean wavelength (Figs. 3F, G).

\section{Distances from pinwheels to the closest OD border}

Pinwheels form in OP maps during the transition between stages one and two, rising in number with developmental time in each map 
and then staying approximately constant during stage two (Fig. 4A). Pinwheels initially form close to the OD column borders and move inwards towards the centre of the OD columns as the OP columns for the non-over-represented orientations become wider (Fig. 4B compared to $4 \mathrm{C}$ ). Stronger over-representations restrict the movement of pinwheels so that they remain closer to OD column borders (Fig. 4C).

\section{Discussion}

In this paper we have used a computational model to make a number of predictions regarding visual map structure under restricted orientation rearing. There is a long history in the field of visual map development of theoretical predictions that have subsequently been tested experimentally. For example, theoretical modelling predicted that the width of ocular dominance columns was dependent on both the extent of lateral connections in the cortex (Swindale, 1980, 1982; Miller et al., 1989) and the degree of correlations in activity between the two eyes (Goodhill and Willshaw, 1990; Goodhill, 1993). These predictions influenced subsequent experimental work, and evidence supporting both predictions has since been obtained (Goodhill, 2007). Dimension-reduction models in particular, most notably the elastic net and Kohonen algorithm, have been shown to produce an excellent match with OP and OD map structure measured experimentally across a wide range of conditions (Erwin et al., 1995; Swindale, 1996; Carreira-Perpiñán et al., 2005; Yu et al., 2005; Farley et al., 2007). The fact that the model we have used in this paper is not new is thus a strength of our approach, since the previous successes of this and related models give greater weight to their predictions in novel cases.

\section{Predicted changes in the $O D$ and $O P$ maps and their implications}

It has been previously demonstrated experimentally, and reproduced by simulation, that in normally reared animals OP contours tend to intersect OD column borders at right angles and OP pinwheels tend to occur at the centre of OD columns (Bartfeld and Grinvald, 1992; Blasdel, 1992b; Obermayer et al., 1992; Obermayer and Blasdel, 1993; Hübener et al., 1997; Kim et al., 1999; Müller et al., 2000; Carreira-Perpiñán et al., 2005). It has also been proposed that the wavelengths of the OD and OP maps are independently controlled and related to each map's developmental time-line (Hoffsümmer et al., 1995, 1996; Goodhill and Cimponeriu, 2000; Wolf et al., 2000;
Giacomantonio and Goodhill, 2007). We found that all of these relationships are challenged by the rearing regime investigated here.

The simulations presented in this paper show that the structure of OD and OP maps, as well as the relationships between them, change as development progresses. After both maps have formed, their development can be divided into two stages based on the appearance of the maps and the relationships between them. Developmental stages are a novel prediction that do not conflict with current experimental evidence. Several studies indicate that in normal animals, OD and OP maps do not form suddenly and simultaneously (Chapman and Bonhoeffer, 1998; Crair et al., 1998; Ruthazer et al., 1999; Crair et al., 2001). In normal cats, OP maps form before OD maps, both over a period of several days (Hubel and Wiesel, 1963; Blakemore and Van Sluyters, 1975; Crair et al., 1998, 2001; but see also Crowley and Katz, 2000; Katz and Crowley, 2002). Chapman and Bonhoeffer (1998) found in ferret that during OP map formation, weakly orientation selective areas, that are initially difficult to distinguish from noise, develop into strongly selective columns. Typically, strongly selective columns are separated by weakly selective areas, which gradually develop orientation selectivity. Once an area becomes strongly orientation selective, it generally does not change OP. Hence, if static OP columns form followed by static OD columns, it is not likely that relationships between maps would change during development in normal animals.

Our simulations predicting stage development do not conflict with the data from normal development because the restricted orientation input forces OP and OD maps to develop at the same time, with OP columns for the over-represented orientations forming earlier than usual, which induces OD column formation. (In fact, because the formation of one map induces the formation of the other soon after, reversing the order of map development does not change our results.) In stage one, cortical points at the borders of the OD/OP columns are weakly orientation selective. The changes in the relationships between maps occur during the transition because OP columns for non-over-represented orientations form in these early border regions, where OD and orientation selectivity is weakest. In our simulations, $\mathrm{OD}$ and $\mathrm{OP}$ are generally static once a preference is formed, in agreement with experiment.

The model predicts that if an OP map contains only the overrepresented orientations (as in stage one), then the OD columns in the same individual will closely match the pattern of OP columns. As a result, the wavelengths of the two maps will be similar and the contours of the two maps will coincide and run parallel. On the other hand, if an OP map contains columns corresponding to the non-over-
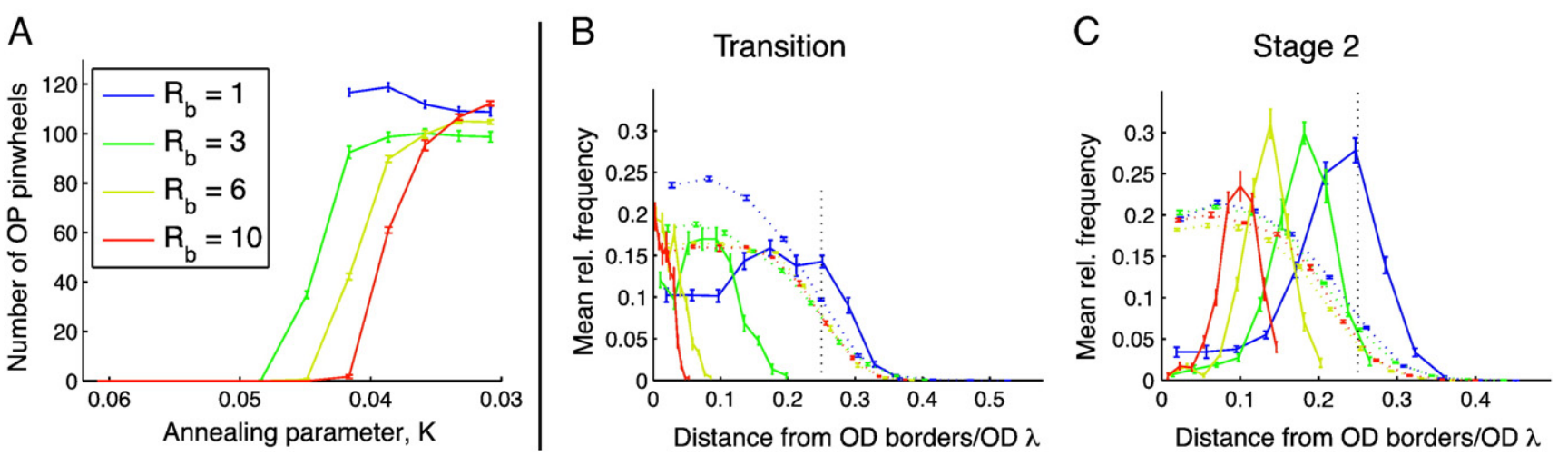

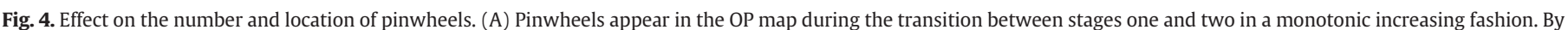

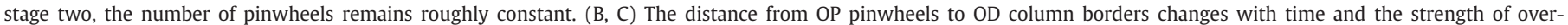

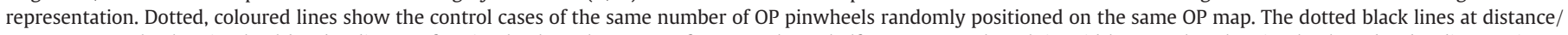

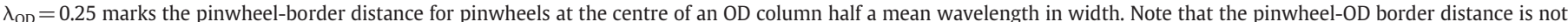

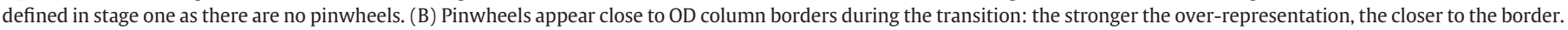

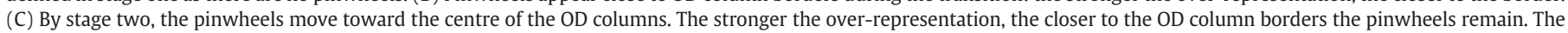
solid, coloured lines are the means of 10 simulations per value of $R_{\mathrm{b}}$. The error bars are the SEM of the data. 
represented orientations, then the layout of the OD map is likely to be related, but not a precise match. In this case, the contours of the two maps are likely to have a tendency to intersect perpendicularly, as occurs in normal animals. In addition, the wavelength of the OP map will be less than that of the OD map, but poorly defined.

In elastic net simulations, the wavelengths of OD and OP maps are normally independently controlled by different parameters, which also dictate the time of development (Hoffsümmer et al., 1995, 1996; Goodhill and Cimponeriu, 2000; Wolf et al., 2000) (although wavelength also depends on the number of maps present (Swindale, 2000, 2004; Carreira-Perpiñán et al., 2005; Farley et al., 2007)). Under the rearing regime investigated here, the wavelengths of the two maps become coupled. They both form earlier than usual, at roughly the same time, and are initially wider than usual. Experimental verification of this prediction using a restricted orientation rearing regime would be evidence in favour of the hypothesis that column width is related to developmental time, as suggested by elastic net simulations here and elsewhere (Goodhill and Cimponeriu, 2000; Giacomantonio and Goodhill, 2007).

The change in the intersection angle of the OP columns with OD column borders is particularly interesting as perpendicular intersection has been suggested as a way to ensure that within each small area of cortex responding to a small area of visual space, a complete set of OP and OD columns are present (Hubel and Wiesel, 1977; Swindale, 1991, 1996, 1998). Good coverage of feature space is essential to prevent perceptual blind spots. The tendency for perpendicular intersection has previously been shown to be remarkably robust under a number of manipulations including experimental strabismus (Löwel et al., 1998) and in dimension-reduction simulations: addition of feature dimensions (Carreira-Perpiñán et al., 2005), monocular deprivation (Carreira-Perpiñán et al., 2005), and angioscotoma representation (Giacomantonio and Goodhill, 2007). Here, we show that later in development, perpendicular intersection can occur even when strongly and directly challenged by the rearing regime.

Under this rearing regime, we found that pinwheels may be found close to OD column borders; the stronger the over-representation, the smaller the distance. Interestingly, pinwheels have previously been shown to remain at the centres of OD columns under monocular deprivation in experiment (Crair et al., 1997b) and simulation (Carreira-Perpiñán et al., 2005). However, under monocular deprivation, the tendency for perpendicular intersection is also maintained (Carreira-Perpiñán et al., 2005). In the restricted orientation rearing regime investigated here, where both relationships cannot be satisfied simultaneously during stage two, perpendicular intersection of OP columns with OD borders is maintained while pinwheels move closer to OD column borders.

\section{Experimentally testing the predictions}

Perhaps the most direct experimental test of these predictions would be to rear cats with permanently implanted goggles fitted with high refractive power spherical or cylindrical lenses (Tanaka et al., 2006), a technique so far unsurpassed in its ability to carefully control visual experience. Intrinsic optical imaging could then be used to measure overall map structure in V1 (Tani and Tanaka, 2008).

Real maps may correspond to simulations terminated in either stage depending on the strength of over-representation and the developmental time-line relative to manipulated rearing. Strengthening the over-representation will increase the likelihood of finding maps in stage one. Because the effect of the manipulation on the maps changes with time, and the time-line of map development may differ between species, we predict that the detailed effect of the manipulation may vary between species and even between individuals of the same species (Giacomantonio and Goodhill, 2007). However, by varying the refractive power of the lenses and/or the length of time for which they are present it should be possible to then titrate the "strength" of over-representation analogously to the parameter $R_{\mathrm{b}}$ in our model, and to investigate the predicted phenomenon of stages of development.

\section{Utility of the restricted orientation rearing regime}

Experimental studies cannot examine what a map in the same individual would look like both with and without a perturbation in rearing condition, and inter-individual map variability makes it difficult to definitively correlate small changes in maps with the manipulation (Löwel, 1994; Rathjen et al., 2002; Kaschube et al., 2003; Adams and Horton, 2006). These experimental limitations have affected the interpretation of results of monocular deprivation and single orientation rearing studies. Experiments have shown that monocular deprivation causes distinct differences in OD but not OP maps (Crair et al., 1997b). It could not be distinguished whether characteristic map relationships were maintained by changing the OD map with a fixed OP map, or coordinated minor changes in the OP map. Similarly, single orientation rearing causes distinct differences in OP but not OD maps, and cannot distinguish whether both maps change in a coordinated manner, or just the OP map (Sengpiel et al., 1999).

Meanwhile, many simulations have predicted that changing inputs causes coordinated changes in multiple feature maps (Swindale, 2000, 2004; Carreira-Perpiñán et al., 2005; Yu et al., 2005). Recently, Farley et al. (2007) provided the first experimental evidence of induced changes to other cortical maps in addition to that which is being directly manipulated. They examined how relationships between maps changed when the OD map was removed in ferret by monocular enucleation. Analysis showed that changes in the remaining feature maps preserved or enhanced particular relationships between the maps. Furthermore, the results matched detailed predictions of a dimension-reduction model. The authors concluded that the relationships between maps are not determined intrinsically, but are instead dictated by mapping the sensory dimensions experienced by the animal according to coverage and continuity principles.

In a similar vein to Farley et al. (2007), the rearing regime presented here extends monocular deprivation and single orientation rearing studies because it causes notable differences in both the OD and OP maps. Furthermore, it challenges the characteristic relationships between maps more strongly than removing the OD map, allowing us to propose distinct signatures that should be present if mapping does indeed occur under the influence of coverage and continuity. Experimental verification of the changes to maps and their relationships predicted here would provide further evidence that the relationships between maps are not intrinsic, and help define the boundaries between activity-dependent and activity-independent aspects of cortical map development. A prominent role for activity in visual development underscores the importance of appropriate visual input during development.

\section{Acknowledgments}

This work was supported by NHMRC grant 525459 to MRI and GJG. CEG is funded by an Australian Postgraduate Award. We thank Miguel Carreira-Perpinann for writing the elastic net simulator on which this work is based, and Rowan Tweedale for helpful comments on the manuscript.

\section{References}

Adams, D.L., Horton, J.C., 2006. Ocular dominance columns in strabismus. Vis. Neurosci. 23, 795-805.

Bartfeld, E., Grinvald, A., 1992. Relationships between orientation-preference pinwheels, cytochrome oxidase blobs, and ocular-dominance columns in primate striate cortex. Proc. Natl. Acad. Sci. U. S. A. 89, 11905-11909.

Blakemore, C., Van Sluyters, R.C., 1975. Innate and environmental factors in the development of the kitten's visual cortex. J. Physiol. 248, 663-716. 
Blasdel, G.G., 1992a. Differential imaging of ocular dominance and orientation selectivity in monkey striate cortex. J. Neurosci. 12, 3115-3138.

Blasdel, G.G., 1992b. Orientation selectivity, preference, and continuity in monkey striate cortex. J. Neurosci. 12, 3139-3161.

Carreira-Perpiñán, M.Á., Goodhill, G.J., 2004. Influence of lateral connections on the structure of cortical maps. J. Neurophysiol. 92, 2947-2959.

Carreira-Perpiñán, M.Á., Lister, R.J., Goodhill, G.J., 2005. A computational model for the development of multiple maps in primary visual cortex. Cereb. Cortex 15, 1222-1233.

Chapman, B., Bonhoeffer, T., 1998. Overrepresentation of horizontal and vertical orientation preferences in developing ferret area 17. Proc. Natl. Acad. Sci. U. S. A. 95 2609-2614.

Crair, M.C., Ruthazer, E.S., Gillespie, D.C., Stryker, M.P., 1997a. Ocular dominance peaks at pinwheel center singularities of the orientation map in cat visual cortex. J. Neurophysiol. 77, 3381-3385.

Crair, M.C., Ruthazer, E.S., Gillespie, D.C., Stryker, M.P., 1997b. Relationship between the ocular dominance and orientation maps in visual cortex of monocularly deprived cats. Neuron 19, 307-318

Crair, M.C., Gillespie, D.C., Stryker, M.P., 1998. The role of visual experience in the development of columns in cat visual cortex. Science 279, 566-570.

Crair, M.C., Horton, J.C., Antonini, A., Stryker, M.P., 2001. Emergence of ocular dominance columns in cat visual cortex by 2 weeks of age. J. Comp. Neurol. 430, 235-249.

Crowley, J.C., Katz, L.C., 2000. Early development of ocular dominance columns. Science 290, 1321-1324.

Dayan, P., 1993. Arbitrary elastic topologies and ocular dominance. Neural Comput. 5 , 392-401.

Durbin, R., Willshaw, D., 1987. An analogue approach to the travelling salesman problem using an elastic net method. Nature 326, 689-691.

Durbin, R., Mitchison, G., 1990. A dimension reduction framework for understanding cortical maps. Nature 343, 644-647.

Erwin, E., Obermayer, K., Schulten, K., 1995. Models of orientation and ocular dominance columns in the visual cortex: a critical comparison. Neural Comput. 7 425-468.

Farley, B.J., Yu, H., Jin, D.Z., Sur, M., 2007. Alteration of visual input results in a coordinated reorganization of multiple visual cortex maps. J. Neurosci. 27, 10299-10310.

Giacomantonio, C.E., Goodhill, G.J., 2007. The effect of angioscotomas on map structure in primary visual cortex. J. Neurosci. 27, 4935-4946.

Goodhill, G.J., 1993. Topography and ocular dominance: a model exploring positive correlations. Biol. Cybern. 69, 109-118.

Goodhill, G.J., 2007. Contributions of theoretical modeling to the understanding of neural map development. Neuron 56, 301-311.

Goodhill, G., Willshaw, D., 1990. Application of the elastic net algorithm to the formation of ocular dominance stripes. Netw.-Comput. Neural Syst. 1, 41-59.

Goodhill, G.J., Willshaw, D.J., 1994. Elastic net model of ocular dominance: overall stripe pattern and monocular deprivation. Neural Comput. 6, 615-621.

Goodhill, G.J., Cimponeriu, A., 2000. Analysis of the elastic net model applied to the formation of ocular dominance and orientation columns. Netw.-Comput. Neural Syst. 11, 153-168.

Goodhill, G.J., Bates, K.R., Montague, P.R., 1997. Influences on the global structure of cortical maps. Proc. R. Soc. B-Biol. Sci. 264, 649-655.

Hirsch, H.V., Spinelli, D.N., 1970. Visual experience modifies distribution of horizontally and vertically oriented receptive fields in cats. Science 168, 869-871.

Hirsch, H.V., Spinelli, D.N., 1971. Modification of the distribution of receptive field orientation in cats by selective visual exposure during development. Exp. Brain Res. $12,509-527$.

Hoffsümmer, F., Wolf, F, Geisel, T., Löwel, S., Schmidt, K, 1995. Sequential bifurcation of orientation-and ocular dominance maps. In: Fogelman-Soulie, F., Gallinari, R. (Eds.), ICANN 95: Proceedings of the international conference on artificial neura networks (Paris), 1. Springer, Berlin, pp. 535-540.

Hoffsümmer, F., Wolf, F., Geisel, T., Löwel, S., Schmidt, K., 1996. Sequential bifurcation and dynamic rearrangement of columnar patterns during cortical development. In: Bower, J.M. (Ed.), Computational neuroscience: trends in research 1995. Academic Press, New York, pp. 197-202.

Hubel, D.H., Wiesel, T.N., 1963. Receptive fields of cells in striate cortex of very young visually inexperienced kittens. J. Neurophysiol. 26, 994-1002.

Hubel, D.H., Wiesel, T.N., 1977. Ferrier lecture. functional architecture of macaque monkey visual cortex. Proc. R. Soc. B-Biol. Sci. 198, 1-59.

Hübener, M., Shoham, D., Grinvald, A., Bonhoeffer, T., 1997. Spatial relationships among three columnar systems in cat area 17. J. Neurosci. 17, 9270-9284.

Kaschube, M., Wolf, F., Geisel, T., Löwel, S., 2002. Genetic influence on quantitative features of neocortical architecture. J. Neurosci. 22, 7206-7217.
Kaschube, M., Wolf, F., Puhlmann, M., Rathjen, S., Schmidt, K.-F., Geisel, T., Löwel, S., 2003. The pattern of ocular dominance columns in cat primary visual cortex: intraand interindividual variability of column spacing and its dependence on genetic background. Eur. J. Neurosci. 18, 3251-3266.

Katz, L.C., Crowley, J.C., 2002. Development of cortical circuits: lessons from ocular dominance columns. Nat. Rev. Neurosci. 3, 34-42.

Kim, D.S., Matsuda, Y., Ohki, K., Ajima, A., Tanaka, S., 1999. Geometrical and topological relationships between multiple functional maps in cat primary visual cortex. Neuroreport 10, 2515-2522.

Löwel, S., 1994. Ocular dominance column development: strabismus changes the spacing of adjacent columns in cat visual cortex. J. Neurosci. 14, 7451-7468.

Löwel, S., Schmidt, K.E., Kim, D.S., Wolf, F., Hoffsümmer, F., Singer, W., Bonhoeffer, T., 1998. The layout of orientation and ocular dominance domains in area 17 of strabismic cats. Eur. J. Neurosci. 10, 2629-2643.

Miller, K.D., Keller, J.B., Stryker, M.P., 1989. Ocular dominance column development: analysis and simulation. Science 245, 605-615.

Mitchell, D.E., Sengpiel, F., 2009. Neural mechanisms of recovery following early visual deprivation. Philos. Trans. R. Soc. B-Biol. Sci. 364, 383-398.

Müller, T., Stetter, M., Hübener, M., Sengpiel, F., Bonhoeffer, T., Gödecke, I., Chapman, B., Löwel, S., Obermayer, K., 2000. An analysis of orientation and ocular dominance patterns in the visual cortex of cats and ferrets. Neural Comput. 12, 2573-2595.

Obermayer, K., Blasdel, G.G., 1993. Geometry of orientation and ocular dominance columns in monkey striate cortex. J. Neurosci. 13, 4114-4129.

Obermayer, K., Blasdel, G.G., Schulten, K., 1992. Statistical-mechanical analysis of selforganization and pattern formation during the development of visual maps. Phys. Rev. A 45, 7568-7589.

Rathjen, S., Schmidt, K.E., Löwel, S., 2002. Two-dimensional analysis of the spacing of ocular dominance columns in normally raised and strabismic kittens. Exp. Brain Res. 145, 158-165.

Ruthazer, E.S., Baker, G.E., Stryker, M.P., 1999. Development and organization of ocular dominance bands in primary visual cortex of the sable ferret. J. Comp. Neurol. 407, 151-165.

Sengpiel, F., Stawinski, P., Bonhoeffer, T., 1999. Influence of experience on orientation maps in cat visual cortex. Nat. Neurosci. 2, 727-732.

Simpson, H.D., Mortimer, D., Goodhill, G.J., 2009. Theoretical models of neural circuit development. Curr. Top. Dev. Biol. 87, 1-51.

Stryker, M.P., Sherk, H., Leventhal, A.G., Hirsch, H.V., 1978. Physiological consequences for the cat's visual cortex of effectively restricting early visual experience with oriented contours. J. Neurophysiol. 41, 896-909.

Swindale, N.V., 1980. A model for the formation of ocular dominance stripes. Proc. R. Soc. B-Biol. Sci. 208, 243-264.

Swindale, N.V., 1982. A model for the formation of orientation columns. Proc. R. Soc. B-Biol. Sci. 215, 211-230.

Swindale, N.V., 1991. Coverage and the design of striate cortex. Biol. Cybern. 65, $415-424$

Swindale, N.V., 1996. The development of topography in the visual cortex: a review of models. Netw.-Comput. Neural Syst. 7, 161-247.

Swindale, N.V., 1998. Cortical organization: modules, polymaps and mosaics. Curr. Biol. 8, R270-R273.

Swindale, N.V., 2000. How many maps are there in visual cortex. Cereb. Cortex 10, 633-643.

Swindale, N.V., 2003. Development of ocular dominance stripes, orientation selectivity, and orientation columns. In: van Ooyen, A. (Ed.), Modeling neural development. MIT Press, Cambridge, Massachusetts, pp. 245-271.

Swindale, N.V., 2004. How different feature spaces may be represented in cortical maps. Netw.-Comput. Neural. Syst. 15, 217-242.

Swindale, N.V., Bauer, H.-U., 1998. Application of Kohonen's self-organizing feature map algorithm to cortical maps of orientation and direction preference. Proc. R. Soc. B-Biol. Sci. 265, 827-838

Tanaka, S., Ribot, J., Imamura, K., Tani, T., 2006. Orientation-restricted continuous visual exposure induces marked reorganization of orientation maps in early life. Neuroimage 30, 462-477.

Tani, T., Tanaka, S., 2008. Interocular imbalance in visual experience affects orientation map formation in cat visual cortex, program no. 769.8/kk14. Society for Neuroscience Annual Meeting, Washington, DC, abstract only. Online.

Wolf, F., Pawelzik, K., Scherf, O., Geisel, T., Löwel, S., 2000. How can squint change the spacing of ocular dominance columns. J. Physiol.-Paris 94, 525-537.

Yu, H., Farley, B.J., Jin, D.Z., Sur, M., 2005. The coordinated mapping of visual space and response features in visual cortex. Neuron 47, 267-280.

Yuille, A.L., Kolodny, J.A., Lee, C.W., 1996. Dimension reduction, generalized deformable models and the development of ocularity and orientation. Neural Netw. 9, 309-319. 